\title{
Model Predictive Control and Generalized Adaptative PID for Load Sharing in Systems of Multiple Sources of Energy
}

\author{
Mouhamadou Falilou Ndiaye ${ }^{1}$, François Guérin², Dimitri Lefebvre ${ }^{2}$ and Papa Alioune Ndiaye ${ }^{1}$ \\ Centre International de Formation et de Recherche en Energie Solaire (CIFRES) - Ecole Supérieure Polytechnique \\ UCAD Dakar, Sénégal BP 5085 Fann $^{1}$ \\ Groupe de Recherche en Electrotechnique et Automatique du Havre (GREAH) Le Havre, 75 rue Bellot, CS80540, \\ 76058 Le Havre Cedex (France) ${ }^{2}$
}

\begin{abstract}
This paper describes the control of the energy transfers in Systems of Multiple Sources of Energy (SMSE). The considered SMSE is a Linear Time Varying (LTV) system in view of the sources and load fluctuations. The proposed control scheme includes DC bus voltage and current regulations loops as well as the automatic adjustment of the power ratio associated with each source. DC bus voltage and current regulations loops are carried out by generalized adaptive PID controllers. The calculation of the power ratios is computed with a Model Predictive Controller (MPC) designed to optimize the system overall performance
\end{abstract}

Keywords Generalized adaptative PID, Model control predictive, energy, multi source.

\section{INTRODUCTION}

In recent years many studies have focused on the control of previously developed by the authors [9]. Indeed, in their Systems of Multiple Sources of Energy (SMSE) [1], [2]. previous works [10] the authors have shown that the duty They are particularly interesting for supplying remote cycle value (DC/DC converter input control) is a variable areas. Indeed, SMSE may constitute the most economical that correlates negatively the available energy and the solution in many applications. In addition, they may result energy required by the load. They have used this property in lower environmental pollution and provide a more to automatically adjust the power ratio of each source reliable supply of electricity through the combination of thanks to a fuzzy logic coordinator [10] and to select the several energy sources. Thus, the development of SMSE best operating modes with an automaton [9].

reduces the probability of energy supply shortage. Furthermore, with the incorporation of energy storage, it also reduces the use of diesel generators (which are commonly required in generation systems based on a single renewable energy source). In this context, SMSEs frequently combine solar and wind energy sources (taking advantage of their complementary nature) with a lead-acid battery bank (to overcome periods of scarce power generation). These studies can be gathered into two major categories: design optimization [3], [4], [5] and control optimization, [6], . In the first approach, the main purpose is to reduce the initial investment capital [5], [6]. In the second approach, various strategies are proposed especially for local optimization, coordination of sources and control of the load [1] [7],[8], [9], [10]. The optimization by accepting a load shedding is also studied [11] as well as the availability on the market of equipment [8] In some cases a shedding not larger than 5\% may impact strongly on the cost (Bilal and al. 2013).

This work belongs to the second category. Usual controllers include fuzzy logic [12], [10], neural network [13], [14], predictive control [15], [16] and multi objective optimization [1]. Furthermore, several works on hierarchical control have suggested the coupling and decoupling of sources [1], [8], [9]. In this paper, a control scheme is proposed based on a hierarchical approach

The first contribution is the construction of a SMSE model controlled by the rate of power. In previous works the model was controlled by an analog control voltage input [10]. The second contribution of this paper is to improve the computation of the power ratio with an MPC controller. This coordinator has to find the optimal values of the power ratio of each source with respect to a performance criterion. It is based on an accurate model which describes the coupling of the sources on the DC bus. The problem is solved as a usual quadratic optimization problem.

The paper is organized as follows: In section II, the topology of the considered SMSE is presented. Section III describes the modeling of sources coupling and presents the local stage of the hierarchical control scheme. The local stage is designed starting from adaptive generalized PID controllers [10] to take into account sources and load fluctuations. The MPC controller which has to compute the optimal values of the power ratios is detailed in section IV. Case studies are simulated and results are presented in section V. Section VI is reserved to the conclusion and perspectives are carried out.

\section{TOPOLOGY OF THE SMSE}

SMSEs that are studied in this paper are made up of heterogeneous power sources coupled on a DC bus 
through DC/DC converters. The supervisory control has been developed [9] for SMSEs with an arbitrary number $\mathrm{N}$ of sources and illustrated for $\mathrm{N}=3$ (Figure 1). For simplicity and portability, SMSEs with a conventional source (for example, a diesel generator, named source 1), a renewable source (for example, solar panels, named source 2) and a storage device (for example, a batteries bank, named source 3) will be considered. Such SMSEs cover a large variety of applications and supply variable loads in single-phase or three-phase alternative form (inverter). Sources 1 and 2 are connected on the DC bus via identical Zero Voltage Switching (ZVS) full bridge isolated Buck converters and source 3 is connected on the DC bus with a reversible Buck Boost DC/DC converter. In Figure 1, I Li stands for the current of source i, $S$ stands for the DC bus voltage, $V_{C i}$ stands for the DC/DC converter i analog voltage control input and $X_{D i}$ stands for the logical value of the contactor that couples source $\mathrm{i}$ on the DC bus. Gains $K_{i}$ will be defined in section III.B.

The considered SMSEs may run in several operating modes [9]. Each operating mode corresponds to a configuration that is characterized by the sources that are coupled on the DC bus. As a consequence, $2^{\mathrm{N}}$ modes will be considered at most for a SMSE with $N$ sources [17]. This supervisory control selects the operating mode for a SMSE with 3 sources and has been fully described by the authors in [9].

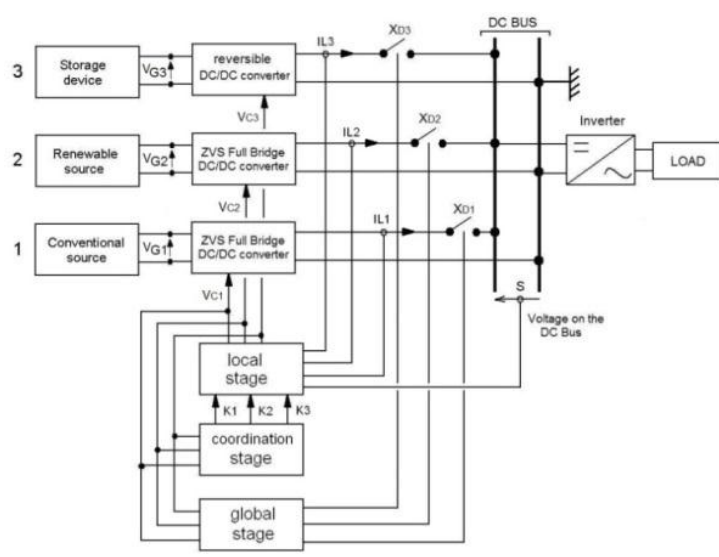

Figure 1. Topology of the SMSE

An automaton (global stage) selects the best operating mode of the SMSE according to several parameters such as the off periods for the renewable source, the state of charge value for the storage source, the charge sustain, and so on. When an operating mode is selected, the local stage must regulate the DC bus voltage and the current provided by each source according to the power ratio provided by the coordinator. It's important to note that the characteristics of the sources are not required for the proposed control design. In this paper, we propose a new coordination stage. It is based on the use of the Model Predictive Control (MPC) and has to compute the optimal values of the power ratio of each source starting from an accurate model which describes the coupling of the sources on the DC bus. To describe this new coordination stage, mode 6 of [9] (conventional and renewable sources are coupled and battery is feed) is considered.

\section{DESIGN OF THE LOCAL STAGE CONTROLLER}

\section{A. Modeling of sources coupling}

The structural diagram of the ZVS full bridge isolated Buck converter is represented on the Figure 2. These DC/DC converters are isolated (HF transformer TR1) Buck converters $\left(D_{5}, D_{6}, D_{7}, D_{8}, L, C_{e}, R_{e}\right)$ with a full bridge $\left(Q_{1}, Q_{2}, Q_{3}, Q_{4}\right)$ and ZVS. The full bridge control $\left(Q_{1}, Q_{2}, Q_{3}, Q_{4}\right)$ is realized by a phase shift controller UC3879 through specialized MOSFET drivers IR2113. The duty cycle value $\square$ is modified by the phase shift between $V a$ and $V b$ voltages. The phase shift is controlled by an analog $\mathrm{DC}$ voltage (between $0 \mathrm{~V}$ and $5 \mathrm{~V}$ ) which represents the DC/DC converter analog voltage control input $\left(V_{C i}\right)$.

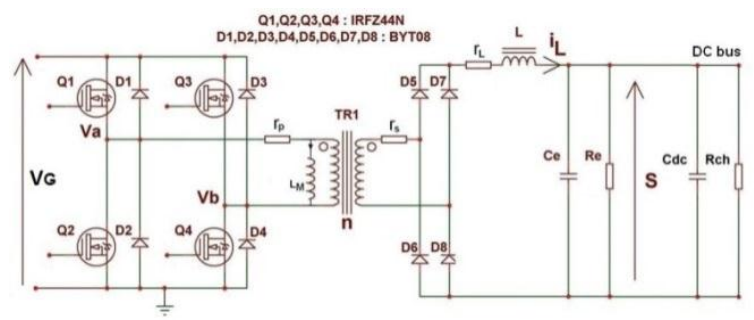

Figure 2. Structural diagram of the ZVS full bridge isolated Buck converter

The partial structural diagram when two identical DC/DC converters are coupled on the DC bus (mode 6) of [9] is represented on the Figure 3.

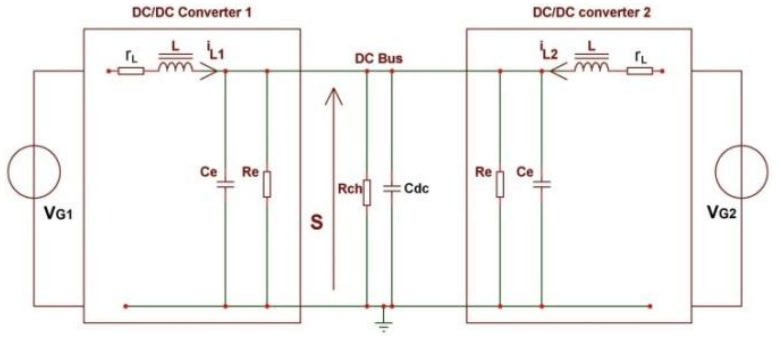

Figure 3. Partial structural diagram of the coupling of two DC/DC converters

State, control and output vectors are respectively defined as $\underline{X}_{M}=\left[I_{L 1}, I_{L 2}, S\right]^{T}, \underline{U}_{M}=\left[V_{C 1}, V_{C 2}\right]^{T}, \underline{Y}_{M}=\left[I_{L 1 m}, I_{L 2 m}, S_{m}\right]^{T}$. The average state space model is written with equation (1):

$$
\begin{aligned}
& \underline{\dot{X}_{M}}=A_{M} X_{M}+B_{M} U_{M} \\
& \Leftrightarrow\left[\begin{array}{c}
\dot{I}_{L 1} \\
\dot{I}_{L 1} \\
\dot{S}
\end{array}\right]=\left[\begin{array}{ccc}
\frac{-r_{L}}{L} & 0 & \frac{-1}{L} \\
0 & \frac{-r_{L}}{L} & \frac{-1}{L} \\
\frac{1}{C_{E Q}} & \frac{1}{C_{E Q}} & \frac{-1}{R_{E Q} \cdot C_{E Q}}
\end{array}\right]\left[\begin{array}{c}
I_{L 1} \\
I_{L 1} \\
S
\end{array}\right]+\left[\begin{array}{cc}
\frac{K_{P S} n V_{G 1}}{L} & 0 \\
0 & \frac{K_{P S} n V_{G 2}}{L} \\
0 & 0
\end{array}\right] \cdot\left[\begin{array}{c}
V_{C 1} \\
V_{C 2}
\end{array}\right] \\
& Y_{M}=C_{M} X_{M} \\
& \Leftrightarrow\left[\begin{array}{c}
I_{L I M} \\
I_{L I M} \\
S_{M}
\end{array}\right]=\left[\begin{array}{ccc}
\mu_{I} & 0 & 0 \\
0 & \mu_{I} & 0 \\
0 & 0 & \mu_{V}
\end{array}\right]\left[\begin{array}{c}
I_{L 1} \\
I_{L 1} \\
S
\end{array}\right]
\end{aligned}
$$

Note that in comparison with our previous works [9], (Guérin and Lefebvre 2013) this average state space model has been simplified. It doesn't take into account the primary resistance $\left(r_{p}\right)$, the secondary resistance $\left(r_{s}\right)$ and 
the magnetizing inductance $\left(L_{M}\right)$ of the HF transformer (TR1) as well as the MOSFET transistors $\left(Q_{1}, Q_{2}, Q_{3}, Q_{4}\right)$ channel resistance $\left(r_{\text {mos }}\right)$. These parameters can be neglected due to their very low values and the estimation of the global losses is not required.

$L, r_{L}, C e$ and $R e$ are respectively the coil inductance, the coil resistance, the capacity and the resistance of the Buck converter. $n$ is the ratio of the HF transformer. $V_{C 1}, V_{C 2}$ are the $\mathrm{DC} / \mathrm{DC}$ converter analog voltage control inputs $(0 \mathrm{~V} / 5 \mathrm{~V}) . V_{G 1}, V_{G 2}$ are the source voltages. $K_{P S}$ is the gain of the phase shift controller UC3879. The duty cycle value is proportional to the analog voltage control input so that $\varphi_{1,2}=1$ when $V_{C l, 2}=5 \mathrm{~V} \quad\left(\varphi_{1,2}=K_{P S} \cdot V_{C l, 2}\right) . R_{C H}$ is the load (supposed to be resistive). It depends on the current provided to the consumers (Icon) supplied through an inverter and eventually to the current used to charge the batteries bank $(I b b)$. Note that a current regulator is used to charge the batteries bank so the current $I b b$ is supposed constant. As a consequence, $R_{C H}$ can be modeled as $R_{C H}=S /(I c o n+I b b)$. Note that for simplicity, no batteries bank will be considered in the following. $R_{E Q}$ is the equivalent resistive load on the DC bus: $R_{E Q}=\left(R_{C H \cdot}(R e / 2)\right) /\left(R_{C H}+(R e / 2)\right) . \quad C d c$ is the DC bus capacity. $C_{E Q}$ is the equivalent capacitor on the DC bus: $C_{E Q}=2 C e+C d c . \mu_{l}, \mu_{V}$ are respectively the gains of the current sensors (LEM-LA55P) and the voltage sensor (LEM-LV25P) associated with their signal conditioners.

\section{B. DC bus voltage and currents regulation loops}

The considered SMSE is a Linear Time Varying (LTV) system in view of the sources and load fluctuations. For this reason, adaptive generalized PID controllers have been investigated to control the $\mathrm{DC} / \mathrm{DC}$ converters under varying operating conditions [10]. The goal is to keep for the voltage and current loops (local stage) the same dynamic behavior (without overshoot or oscillations) whatever the sources and load fluctuations, when the power ratio is modified by the coordination stage.

Under the constraint that the DC bus voltage is constant, it is possible to drive the power delivered to the load by controlling the current provided by each source (load sharing). The cascaded controller (Figure 4) has been designed starting from the average state space model (1) and has been fully described in [10]. It regulates the DC bus voltage (whatever the sources and load fluctuations) and the current provided by each source. It also includes adjustable (between $0 \%$ and $100 \%)$ gains $\left(K_{1}, K_{2}\right)$ making it possible to control the power ratio provided by each source.

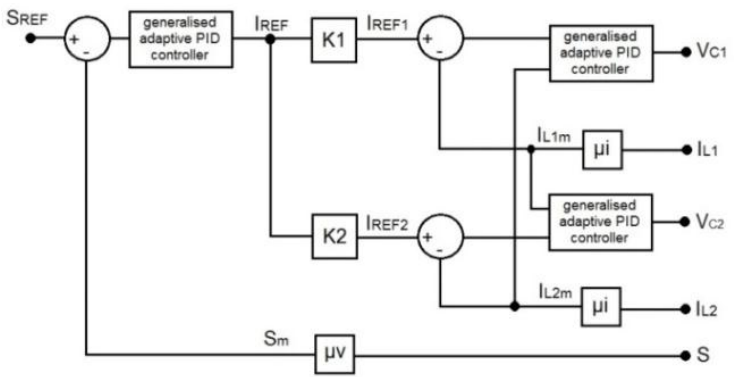

Figure 4. Local stage controller
The dynamic behavior of each loop (current and voltage) can be described [10] with first order closed loop transfer functions according to equations (2) to (4):

$$
\begin{gathered}
I_{L 1}(s)=\frac{1 / \mu_{I}}{1+\frac{1}{H_{1} \cdot K_{C 1} \cdot \mu_{I}} s} \cdot I_{R E F 1}(s)=\frac{1 / \mu_{I}}{1+\tau_{I} \cdot s} \cdot I_{R E F 1}(s) \\
I_{L 2}(s)=\frac{1 / \mu_{I}}{1+\frac{1}{H_{2} \cdot K_{C 2} \cdot \mu_{I}} s} \cdot I_{R E F 2}(s)=\frac{1 / \mu_{I}}{1+\tau_{I} \cdot s} \cdot I_{R E F 2}(s) \\
S(s)=\frac{1 / \mu_{V}}{1+\frac{\mu_{I}}{K_{V} \cdot R_{E Q} \cdot \mu_{V}} s} \cdot S_{R E F}(s)=\frac{1 / \mu_{V}}{1+\tau_{V} \cdot s} \cdot S_{R E F}(s) \\
I_{R E F 1}(s)=K_{1} \cdot I_{R E F}(s) \quad I_{R E F 2}(s)=K_{2} \cdot I_{R E F}(s) \\
I_{R E F}(s)=\frac{\mu_{I} \cdot\left(1+\tau_{I} \cdot s\right)}{R_{E Q}} \cdot S(s)
\end{gathered}
$$

with: $K_{1}+K_{2}=1$ and $K_{1}>0, K_{2}>0$

$I_{R E F 1}, I_{R E F 2}$ and $S_{R E F}$ are respectively the reference values of the current and voltage loops. $\tau_{I}$ is the desired time constant for the current loops. $\tau_{V}$ is the desired time constant for the voltage loop (DC bus). Note that if we neglect the loss of the ZVS full bridge DC/DC converters, the duty cycle values $\left(\varphi_{1}, \varphi_{2}\right)$ can be easily estimated [9] thanks to the following equations:

$$
S(s)=n \cdot \varphi_{1}(s) \cdot V_{G 1}(s) \quad S(s)=n \cdot \varphi_{2}(s) \cdot V_{G 2}(s)
$$

In equation (7), the output voltage $(S)$ is the same for both DC/DC converters since they are coupled on the same DC bus. To conclude, the proposed cascaded adaptive controller doesn't have steady state errors (for both currents and DC bus voltage) and will keep the desired dynamic behavior $\left(\tau_{\mathrm{I}}, \tau_{\mathrm{V}}\right)$ whatever the sources and load fluctuations when the power ratios $\left(K_{1}, K_{2}\right)$ will be modified by the coordination stage (load sharing).

\section{DESIGN OF THE COORDINATION STAGE CONTROLLER}

The coordination stage is based on a Model Predictive Control (MPC) approach. The paradigm of MPC is the use of an internal model to predict the behavior of the system and choose the best decision according to a cost criteria while respecting the functional constraints [18]. MPC is widely used in the field of energy [16]. In the field of the SMSEs it is already used to optimize the production [12] and for supervision control issues [19], [15]. Our approach is based on the use of the duty cycle values of the DC/DC converters $\left(\varphi_{1}, \varphi_{2}\right)$ as an indirect measurement of the available energy. Indeed, the duty cycle value is a variable that correlates negatively the available energy and the energy required by the load. Indeed, to regulate the DC bus voltage and the current provided by each source, the cascaded adaptive controller previously described, makes increasing the duty cycle values (through VC1, VC2) when the load increases or when the source voltage decreases and inversely [9]. Consequently additional external sensors are not required to estimate the available energy. 


\section{Design of the internal model}

The MPC controller (coordination stage) has to compute the optimal values of the power ratios $(\mathrm{K} 1, \mathrm{~K} 2)$. To do that, we need to find an internal model in which the power ratios $(\mathrm{K} 1, \mathrm{~K} 2)$ belong to the input vector and the duty cycle values $(\varphi 1, \varphi 2)$ to the output vector. Over the horizon of prediction, we assume that the DC bus voltage $(S)$ is constant and equal to SREF (the voltage regulation loop doesn't have steady state error). Thus, equation (6) can be rewritten according to the following equation:

$$
\mu_{I} \cdot S_{R E F}(s)=R_{E Q} \cdot I_{R E F}(s)
$$

Starting from equations (2) to (8), the internal model can be written according to equation (12). State, control and output vectors are respectively defined as:

$$
\begin{aligned}
& \mathrm{X}=[\mathrm{IL} 1, \mathrm{IL} 2, \mathrm{~S}] \mathrm{T} \\
& \mathrm{U}=[\mathrm{K} 1, \mathrm{~K} 2] \mathrm{T} \\
& \mathrm{Y}=[\mathrm{IL} 1, \mathrm{IL} 2, \mathrm{~S}, \varphi 1, \varphi 2] \mathrm{T} \\
& \underline{\dot{X}}=A \underline{X}+B \underline{U} \\
& \Leftrightarrow\left[\begin{array}{c}
\dot{I}_{L 1} \\
\dot{I}_{L 1} \\
\dot{S}
\end{array}\right]=\left[\begin{array}{ccc}
-\frac{1}{\tau_{\mathrm{i}}} & 0 & 0 \\
0 & -\frac{1}{\tau_{\mathrm{i}}} & 0 \\
0 & 0 & -\frac{1}{\tau_{\mathrm{V}}}
\end{array}\right]\left[\begin{array}{c}
I_{L 1} \\
I_{L 2} \\
S
\end{array}\right]+\left[\begin{array}{cc}
\frac{\mathrm{S}_{\mathrm{REF}}}{\mathrm{R}_{\mathrm{EQ}} \tau_{I}} & 0 \\
0 & \frac{\mathrm{S}_{\mathrm{REF}}}{\mathrm{R}_{\mathrm{EQ}} \tau_{\tau_{l}}} \\
\frac{\mathrm{S}_{\mathrm{REF}}}{\tau_{V} \mathrm{v}} & \frac{\mathrm{S}_{\mathrm{REF}}}{\tau_{V} \mu_{\mathrm{v}}}
\end{array}\right] \cdot\left[\begin{array}{l}
K_{1} \\
K_{2}
\end{array}\right] \\
& \underline{Y}=C \underline{X}+D \underline{U} \\
& \Leftrightarrow\left[\begin{array}{c}
I_{L 1} \\
I_{L 1} \\
S \\
\varphi_{1} \\
\varphi_{2}
\end{array}\right]=\left[\begin{array}{ccc}
1 & 0 & 0 \\
0 & 1 & 0 \\
0 & 0 & 1 \\
0 & 0 & \frac{1}{n V_{G 1}} \\
0 & 0 & \frac{1}{n V_{G 2}}
\end{array}\right]\left[\begin{array}{c}
I_{L 1} \\
I_{L 2} \\
S
\end{array}\right] \quad D=0
\end{aligned}
$$

In discrete time (sampling period Te), equation (12) is rewritten according to a first order approximation:

$$
\begin{gathered}
X_{k+1}=F X_{k}+G U_{k} \\
Y_{k}=\mathrm{C} \cdot X_{k}+D U_{k}
\end{gathered}
$$

with: $F=I+A . T e$ and $G=B . T e$

I is the identity matrix of appropriate dimensions.

B. Formulation of the cost criteria

The previous internal model is used to predict the behavior of the system and choose the best decision according to a cost criteria while respecting the functional constraints. The cost criteria $(\mathrm{J})$ to minimize is the distance between the reference output YREF and output Y:

$J_{k}=\sum_{j=1}^{N}\left(Y_{k+j}-Y_{R E F}\right)^{T} \cdot\left(Y_{k+j}-Y_{R E F}\right)$

Where $\mathrm{N}$ is the horizon of prediction. The desired output vector $\mathrm{YREF}=(\mathrm{IL} 1 \mathrm{REF}$ IL2REF SREF $\varphi 1 \mathrm{REF} \varphi 2 \mathrm{REF}) \mathrm{T}$ is selected according to technical considerations.

Let us assume that the sources, the load, and the control vector remain constant over the horizon of prediction, the system output $\mathrm{Y}(12)$ is predicted over N steps with the following equations:

$$
\begin{gathered}
X_{k+n}=F^{n} \cdot X_{k}+\left(\sum_{j=0}^{j=n-1} F^{j}\right) \cdot G \cdot U_{k} \\
Y_{k+n}=C \cdot F^{n} \cdot X_{k}+\left[C \cdot\left(\sum_{j=0}^{j=n-1} F^{j}\right) \cdot G+D\right] \cdot U_{k}
\end{gathered}
$$

After having remove all constant terms (with respect to $\mathrm{Uk}$ ), the expression of the cost criteria is rewritten as:

$$
J_{k+n}=\frac{1}{2} U_{k}^{T} H_{k+n} U_{k}+f_{k+n} U_{k}
$$

with:

$$
\begin{gathered}
H_{k+n}=2 \cdot\left[C \cdot\left(\sum_{j=0}^{j=n-1} F^{j}\right) \cdot G+D\right] \cdot\left[C \cdot\left(\sum_{j=0}^{j=n-1} F^{j}\right) \cdot G+D\right]^{T} \\
f_{k+n}=\left[Y_{k}^{T}\left(C \cdot F^{n}\right)^{T}-Y_{r e f}^{T}\right] \cdot\left[C \cdot\left(\sum_{j=0}^{j=n-1} F^{j}\right) \cdot G+D\right]
\end{gathered}
$$

that leads to a quadratic cost criteria (with respect to Uk):

$$
J_{k}=\frac{1}{2} \sum_{j=1}^{N}\left(U_{k}^{T} H_{k+j} U_{k}+f_{k+j} \cdot U_{k}\right)
$$

The cost criteria ( $\mathrm{J})$ is minimized (with respect to $\mathrm{Uk}$ ) by using a standard quadratic optimization method. The following constraints are required by the load sharing:

- $\mathrm{K} 1+\mathrm{K} 2=1$

- $0<\mathrm{Ki}$

- $\mathrm{Ki}<1$

\section{SIMULATIONS AND RESULTS}

To highlight the performances of the controllers (local and coordination stages), several Matlab/Simulink simulations have been done with the following parameters: $\mathrm{L}=120 \mu \mathrm{H}$, $\mathrm{rL}=0.5 \Omega, \quad \mathrm{Ce}=330 \mu \mathrm{F}, \quad \mathrm{Re}=56 \mathrm{~K} \Omega, \quad \mathrm{Cdc}=1 \mu \mathrm{F}, \quad \mathrm{n}=10$, $\mu \mathrm{I}=1 \mathrm{~V} / \mathrm{A}, \quad \mu \mathrm{v}=6.25 \mathrm{mV} / \mathrm{V}, \quad \mathrm{KPS}=0.2 \mathrm{~V}-1 . \quad$ In addition, $\mathrm{Te}=0.15 \mathrm{~ms}$ is the sampling period. For the current and voltage regulation loops the desired time constants are respectively $\tau \mathrm{i}=20 \mathrm{~ms}, \tau \mathrm{V}=200 \mathrm{~ms}$. The load is supposed constant: $\mathrm{RCH}=30 \Omega$ ( $\mathrm{Ibb}=0 \mathrm{~A}$, Icon $=10 \mathrm{~A}$ for example).

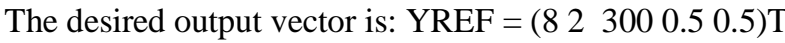

Notes:

- $\mathrm{SREF}=300 \mathrm{~V}$ is the desired DC bus voltage.

- $\varphi 1,2 \mathrm{REF}=0.5$ corresponds to duty cycle values chosen (running point) in the middle of the range (phase shift controller UC3879).

The source voltages (VG1,VG2) are represented in Figure 5 . They varies between the maximum $(120 \mathrm{~V})$ and minimal $(30 \mathrm{~V})$ values tolerated by the DC/DC converter. The adaptive generalized PID adjust the duty cycles values (Figure 6) in order to regulate the DC bus voltage (Figure 7) and the current provided to the load by each source (Figure 10). One can notice that the variations of the power ratio and the source voltages do not disturb the regulation loops.

The MPC calculates the optimal power ratios $\mathrm{K} 1$ and $\mathrm{K} 2$ (Figure 9). The power ratios are automatically calculated 
and adjusted at each time step. The profile of each source current (Figure 10) is similar to the profile of the power ratios (Figure 9). The sum of the source currents (Figure 10 ) is equal to the current required by the load (Figure 8).

In this work we simulated the situation where the source voltages vary in normal operating conditions. Sometimes the source voltage 2 is the double of the source voltage 1 (for example, at time instant 5s).

We can see that the MPC controller calculate the best power ratios in order to keep the duty cycle values near to 0.5 . It always maintain them between 0.2 and 0.8 . This property is important. Indeed the authors [10] were shown that the available energy from the source is function of duty cycle. For any variation, the system can be adjusted

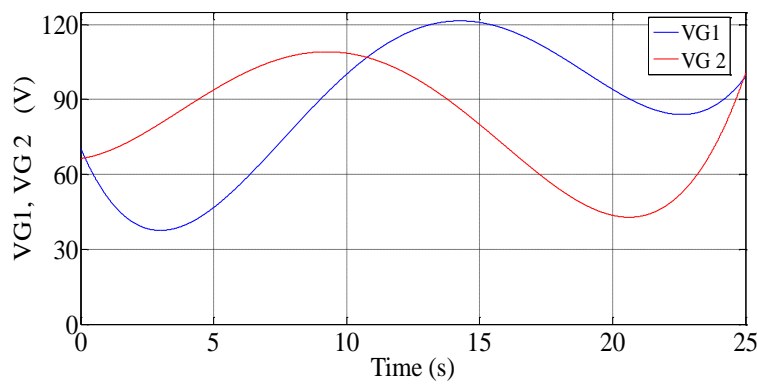

Figure 5. Source voltages

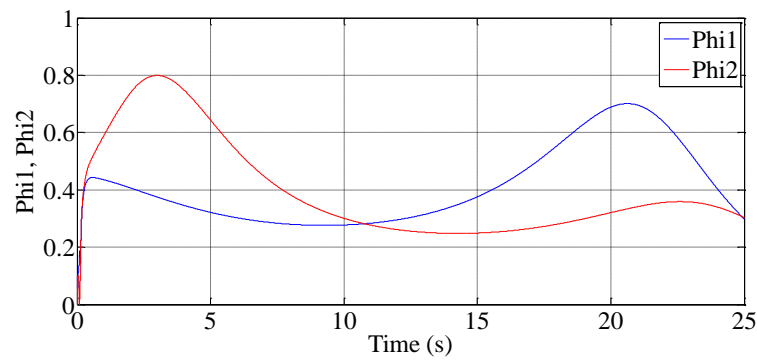

Figure 6. Duty cycle values

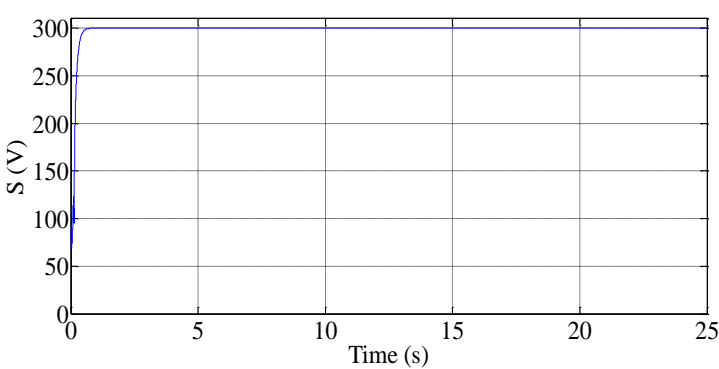

Figure 7. DC Bus voltage

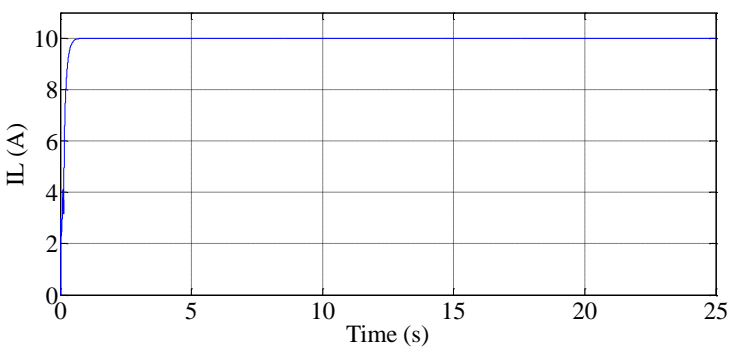

Figure 8. Load current

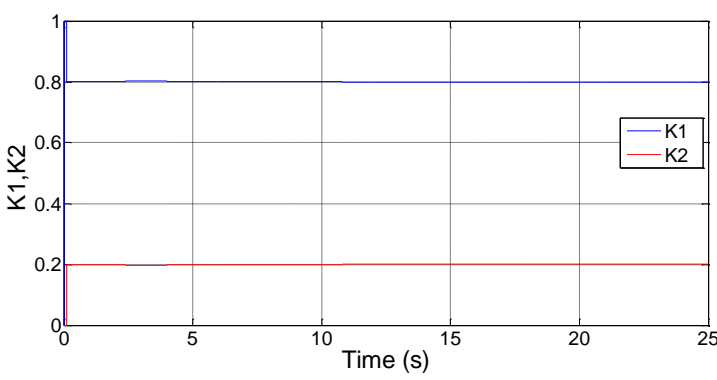

Figure 9. Power ratios

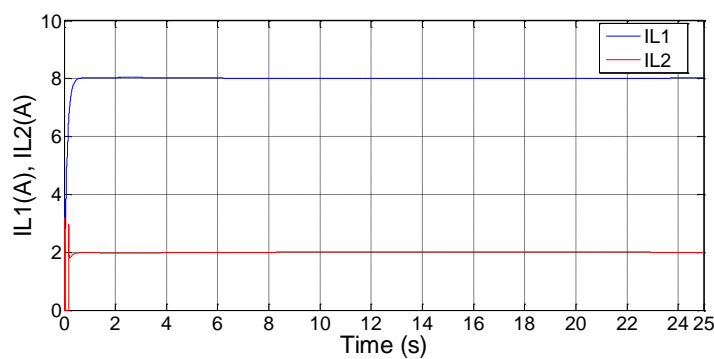

Figure 10. Currents provided by each source

\section{CONCLUSION AND PERSPECTIVES}

In this paper, a control scheme that includes adaptive generalized PID and model predictive control has been proposed to improve the load sharing in SMSEs. The local controllers cooperate with a MPC coordinator that tunes the power ratio provided by each source according to the load and duty cycle estimations. Compared to our previous work [10], the MPC coordinator compute the optimal values of the power ratios by taking into account technical considerations and functional constraints. We can note that additional sensors and external information are not required to estimate the energy available. The performance of the system ensures a reserve to respond to the unexpected power variations. In our future works, both controllers (local stage and coordination stage) will be implemented into a Microchip dsPIC30F6010A microcontroller with industrial DC/DC converters. We will also improve the cost criteria to take into account economical and environmental performance. Our main efforts will finally be devoted on the supervised control with automata in order to find the optimal configurations according to the climatic conditions.

\section{REFERENCES}

[1] R. Baños, F. Manzano-Agugliaro, F. G. Montoya, C. Gil, A. Alcayde, and J. Gómez, 'Optimization methods applied to renewable and sustainable energy: A review', Renew. Sustain. Energy Rev., vol. 15, no. 4, pp. 1753-1766, May 2011.

[2] M. Iqbal, M. Azam, M. Naeem, A. S. Khwaja, and A. Anpalagan, 'Optimization classification, algorithms and tools for renewable energy: A review', Renew. Sustain. Energy Rev., vol. 39, pp. 640654, Nov. 2014

[3] B. O. Bilal, V. Sambou, C. M. . Kébé, P. A. Ndiaye, and M. Ndongo, 'Methodology to Size an Optimal Stand-Alone PV/wind/diesel/battery System Minimizing the Levelized cost of Energy and the CO2 Emissions', Energy Procedia, vol. 14, pp. 1636-1647, 2012

[4] O. Hafez and K. Bhattacharya, 'Optimal planning and design of a renewable energy based supply system for microgrids', Renew. Energy, vol. 45, pp. 7-15, Sep. 2012. 
[5] A. Maleki and A. Askarzadeh, 'Optimal sizing of a PV/wind/diesel system with battery storage for electrification to an off-grid remote region: A case study of Rafsanjan, Iran', Sustain. Energy Technol. Assess., vol. 7, pp. 147-153, Sep. 2014.

[6] A. T. D. Perera, R. A. Attalage, K. K. C. K. Perera, and V. P. C. Dassanayake, 'Designing standalone hybrid energy systems minimizing initial investment, life cycle cost and pollutant emission', Energy, vol. 54, pp. 220-230, Jun. 2013.

[7] B. O. Bilal, V. Sambou, P. A. Ndiaye, C. M. F. Kébé, and M. Ndongo, 'Study of the Influence of Load Profile Variation on the Optimal Sizing of a Standalone Hybrid PV/Wind/Battery/Diesel System', Energy Procedia, vol. 36, pp. 1265-1275, 2013.

[8] R. Belfkira, L. Zhang, and G. Barakat, 'Optimal sizing study of hybrid wind/PV/diesel power generation unit', Sol. Energy, vol. 85, no. 1, pp. 100-110, Jan. 2011.

[9] F. Guerin, D. Lefebvre, and V. Loisel, 'Supervisory control design for systems of multiple sources of energy', Control Eng. Pract., vol. 20, no. 12, pp. 1310-1324, Dec. 2012.

[10] F. Guérin and D. Lefebvre, 'Adaptive generalized PID controllers and fuzzy logic coordinator for load sharing in SMSE', in Decision and Control (CDC), 2013 IEEE 52nd Annual Conference on, 2013, pp. 5588-5593.

[11] M. S. Carmeli, F. Castelli-Dezza, M. Mauri, G. Marchegiani, and D. Rosati, 'Control strategies and configurations of hybrid distributed generation systems', Renew. Energy, vol. 41, pp. 294-305, May 2012.

[12] M. Sarailoo, Z. Rahmani, and B. Rezaie, 'Fuzzy Predictive Control of Step-Down DC-DC Converter Based on Hybrid System Approach', Int. J. Intell. Syst. Appl., vol. 6, no. 2, pp. 1-13, Jan. 2014.

[13] K. Karabacak and N. Cetin, 'Artificial neural networks for controlling wind-PV power systems: A review', Renew. Sustain. Energy Rev., vol. 29, pp. 804-827, Jan. 2014.

[14] N. A., T. L., S. G., and S. F. S., 'Development of a multilayer perceptron (MLP) based neural network controller for grid connected photovoltaic system', Int. J. Phys. Sci., vol. 9, no. 3, pp. 41-47, Feb. 2014.

[15] W. Qi, J. Liu, X. Chen, and P. D. Christofides, 'Supervisory Predictive Control of Standalone Wind/Solar Energy Generation Systems', IEEE Trans. Control Syst. Technol., vol. 19, no. 1, pp. 199-207, Jan. 2011.

[16] A. M. Kassem, 'Robust voltage control of a stand alone wind energy conversion system based on functional model predictive approach', Int. J. Electr. Power Energy Syst., vol. 41, no. 1, pp. 124-132, Oct. 2012.

[17] A. Badara Mboup, F. Guerin, D. Lefebvre, and P. Alioune Ndiaye, 'Control design for multisource systems based on DC/DC converters duty cycle value', COMPEL - Int. J. Comput. Math. Electr. Electron. Eng., vol. 30, no. 1, pp. 310-335, Jan. 2011.

[18] J. Richalet, G. Lavielle, J. Mallet, and G. Dreyfus, La commande prédictive. Paris: Eyrolles, 2004

[19] M. Khalid and A. V. Savkin, 'A model predictive control approach to the problem of wind power smoothing with controlled battery storage', Renew. Energy, vol. 35, no. 7, pp. 1520-1526, Jul. 2010. 